\title{
Reverse engineering a spectrum: using fluorescent spectra of molecular hydrogen to recreate the missing Lyman- $\alpha$ line of pre-main sequence stars
}

\author{
J L Linsky ${ }^{1}$, G Herczeg ${ }^{2}$ and B E Wood ${ }^{3}$ \\ ${ }^{1}$ JILA, University of Colorado and NIST, Boulder, CO 80309-0440, USA \\ ${ }^{2}$ California Institute of Technology, Pasadena, CA 91125, USA \\ ${ }^{3}$ Naval Research Laboratory, Space Science Division, Code 7660, Washington, DC 20375, USA \\ E-mail: jlinsky@jila.colorado.edu
}

Received 17 September 2008

Accepted for publication 19 September 2008

Published 19 December 2008

Online at stacks.iop.org/PhysScr/T133/014018

\begin{abstract}
The hydrogen Lyman- $\alpha$ (Ly $\alpha)$ line, a major source of ionization of metals in the circumstellar disks of pre-main sequence (PMS) stars, is usually not observed due to absorption by interstellar and circumstellar hydrogen. We have developed a technique to reconstruct the intrinsic Ly $\alpha$ line using the observed emission in the $\mathrm{H}_{2}$ B-X lines that are fluoresced by Ly $\alpha$. We describe this technique and the subsequent analysis of the ultraviolet (UV) spectra of the TW Hya, RU Lupi and other PMS stars. We find that the reconstructed Ly $\alpha$ lines are indeed far brighter than any other feature in the UV spectra of these stars and therefore play an important role in the ionization and heating of the outer layers of circumstellar disks.
\end{abstract}

(Some figures in this article are in colour only in the electronic version.)

\section{From the observed $\mathrm{H}_{2}$ spectrum to the properties of the $\mathrm{H}_{2}$ gas in the disk of TW Hya}

Pre-main sequence (PMS) stars exhibit rich ultraviolet (UV) spectra containing many emission lines formed in their chromospheres, circumstellar disks, accretion shocks and jets. In their survey of PMS stars with the International Ultraviolet Explorer satellite, Johns-Krull et al (2000) and Valenti et al (2000) noted that emission lines of $\mathrm{H}_{2}$ are prominent in the spectra of many of these stars. We have followed up on this survey with higher resolution spectra of PMS stars obtained by the Space Telescope Imaging Spectrograph (STIS) instrument on the Hubble Space Telescope (HST) beginning with the nearest classical T Tauri star, TW Hya. Figure 1 (from Herczeg et al (2002)) shows a small portion of this TW Hya spectrum. In the complete STIS spectrum, $\mathrm{H}_{2}$ emission lines are detected between 1202 and $1636 \AA$. Other $\mathrm{H}_{2}$ emission lines are detected at shortened wavelengths in spectra obtained by the Far Ultraviolet Spectrograph Explorer (FUSE) spacecraft.
We found that 194 of the emission lines observed by STIS are transitions from 25 levels in the $\mathrm{H}_{2}$ B electronic state to vibration-rotation levels in the ground electronic state. Herczeg et al (2006) found that 22 of the 25 levels in the $\mathrm{B}$ electronic state are populated by radiative transitions from excited levels in the ground electronic state (see figure 2) at wavelengths between 1212.426 and $1219.368 \AA$ that is within the broad Lyman- $\alpha(\operatorname{Ly} \alpha)$ emission line. Three of these levels are populated by radiation at $1548.146 \AA$, which is coincident with the $\mathrm{C}$ IV emission line. Thus, Ly $\alpha$ pumping is responsible for nearly all of this fluorescent $\mathrm{H}_{2}$ spectrum. Figure 3 shows the wavelengths of these pumping transitions compared with the observed Ly $\alpha$ line.

Figure 3 clearly shows that much of the intrinsic Ly $\alpha$ emission line is not detected due to interstellar and circumstellar Ly $\alpha$ absorption. Despite this strong absorption, the observed Ly $\alpha$ line is by far the brightest feature in TW Hya's UV spectrum and is responsible for the observed fluorescent $\mathrm{H}_{2}$ emission. Since the radial velocity of the $\mathrm{H}_{2}$ gas is the same as the photosphere for this edge-on disk 


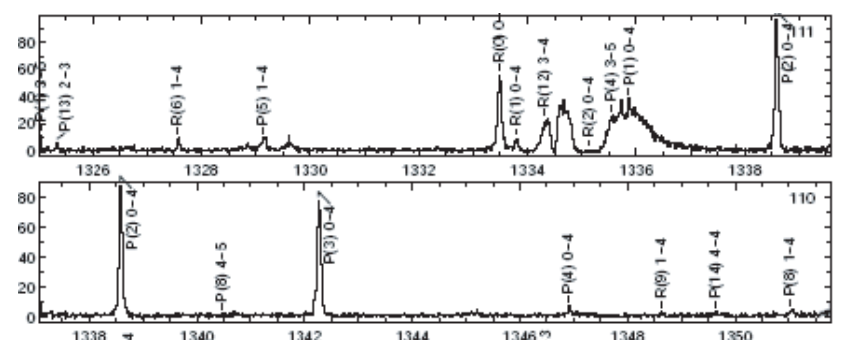

Figure 1. A portion of the HST/STIS E140M spectrum of TW Hya. Lyman-band transitions of $\mathrm{H}_{2}$ that are pumped by Ly $\alpha$ are labeled. Some of these transitions are too weak to be detected. A total of 146 fluorescent $\mathrm{H}_{2}$ lines pumped by Ly $\alpha$ are observed in the HST/STIS spectrum. See Herczeg et al (2002).

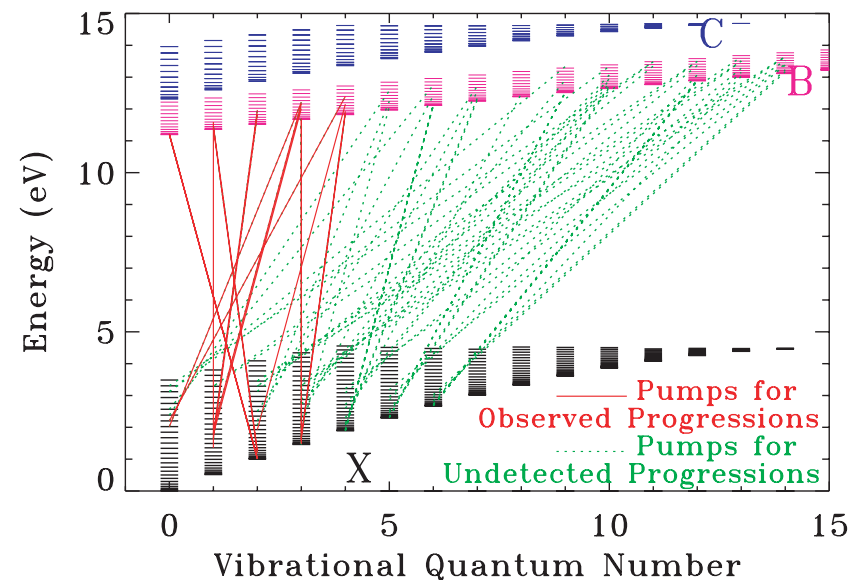

Figure 2. Energy levels calculated by Abgrall et al (1993) for the ground $(\mathrm{X})$ and excited (B and $\mathrm{C}$ ) electronic levels of $\mathrm{H}_{2}$. Lyman band transitions $(\mathrm{B}-\mathrm{X})$ typically occur in the UV range observed by HST/STIS, while Werner band $(\mathrm{C}-\mathrm{X})$ transitions occur in the far-UV (FUV) range observed by FUSE. Solid lines indicate transitions pumped by Ly $\alpha$ leading to observed fluorescent emission lines. Dashed lines indicate transitions that can be pumped by Ly $\alpha$ but do not lead to detected fluorescent emission. Note that the levels in the ground electronic state have vibration-rotation energies as large as $4.2 \mathrm{eV}$, indicating that the $\mathrm{H}_{2}$ gas in the disk is warm (about $2500 \mathrm{~K}$ ), but the highest energy levels may be non-thermal. See Herczeg et al (2002).

system, the $\mathrm{H}_{2}$ gas is most likely located in the circumstellar disk. Thus, an accurate determination of the intrinsic Ly $\alpha$ emission line flux and profile is needed to infer the physical conditions in the disk. We therefore need to reconstruct, that is reverse engineer, the Ly $\alpha$ emission line for TW Hya and other PMS stars.

\section{Reconstructing the $\operatorname{Ly} \alpha$ line profile and flux}

We use the observed fluorescent $\mathrm{H}_{2}$ lines to reconstruct the Ly $\alpha$ emission at each pumping wavelength by assuming that the $\mathrm{H}_{2}$ gas resides in a warm, plane-parallel slab, following the procedure described by Herczeg et al (2004). The total emission from an $\mathrm{H}_{2}$ upper level, $F_{\mathrm{H}_{2}}$, is proportional to the oscillator strength, $f$, of the pumping transition, the population of $\mathrm{H}_{2}$ in the lower level, $N_{\mathrm{L}}\left(\mathrm{H}_{2}\right)$, which depends

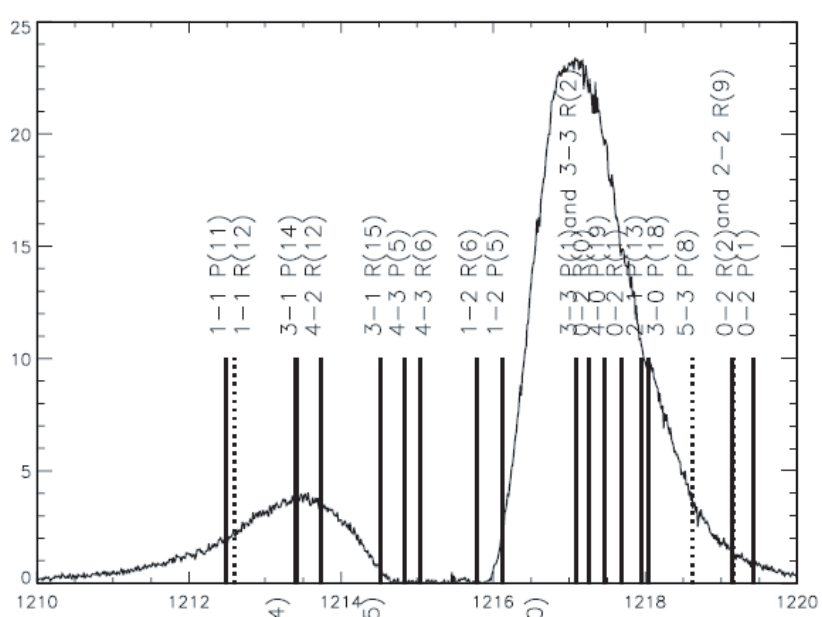

Figure 3. HST/STIS E140M spectrum of the Ly $\alpha$ line of TW Hya. The solid lines indicate pumping transitions with definite detections of fluorescent $\mathrm{H}_{2}$ emission lines. Dashed lines indicate transitions where the fluorescent lines are tentatively detected. See Herczeg et al (2002).

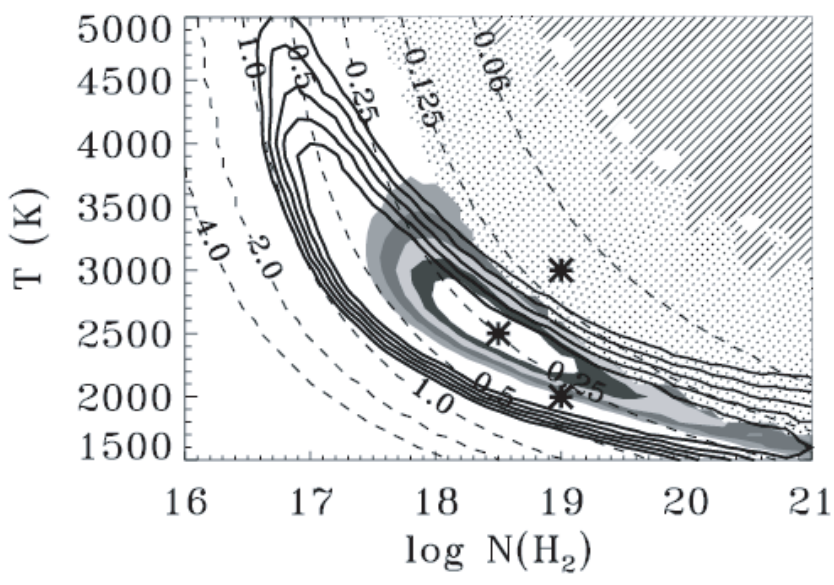

Figure 4. Inferred physical properties of the $\mathrm{H}_{2}$ gas in the disk of TW Hya based on pumping by the reconstructed Ly $\alpha$ line. Shaded regions show the $1-5 \sigma$ areas of $\mathrm{T}-\mathrm{N}\left(\mathrm{H}_{2}\right)$ space allowed by errors in the reconstructed Ly $\alpha$ line and the solid lines indicate the $1-5 \sigma$ contours for the fits to the $\mathrm{H}_{2}$ lines. Dots indicate the parameter space that we rule out because those parameters predict that lines pumped by 7-4 $\mathrm{P}(5)$ should be observed, and the diagonal lines indicate the parameter space ruled out because many additional other progressions should be observed but are not detected. Filling factor contours (dashed lines) of 0.125-4.0 are also shown. A filling factor above 1 is unphysical. Three models with acceptable reconstructed Ly profiles are shown as asterisks. See Herczeg et al (2004).

on the temperature and column density of the slab, the strength of Ly $\alpha$ emission at the pumping wavelength, $F_{\text {Ly } \alpha}$, and the solid angle filling factor, $\eta$, subtended by the $\mathrm{H}_{2}$ gas as seen from the Ly $\alpha$ emission source. We calculate a Voigt absorption profile for each pumping transition. The Ly $\alpha$ flux $F_{\text {Ly } \alpha}$ at the pumping wavelength is given by

$$
F_{\mathrm{Ly} \alpha}=\frac{F_{\mathrm{H}_{2}} / f \eta N_{L}\left(\mathrm{H}_{2}\right)}{\int_{\lambda}\left[1-\mathrm{e}^{-\tau_{\lambda}\left(T, N\left(\mathrm{H}_{2}\right)\right)}\right] \mathrm{d} \lambda} .
$$




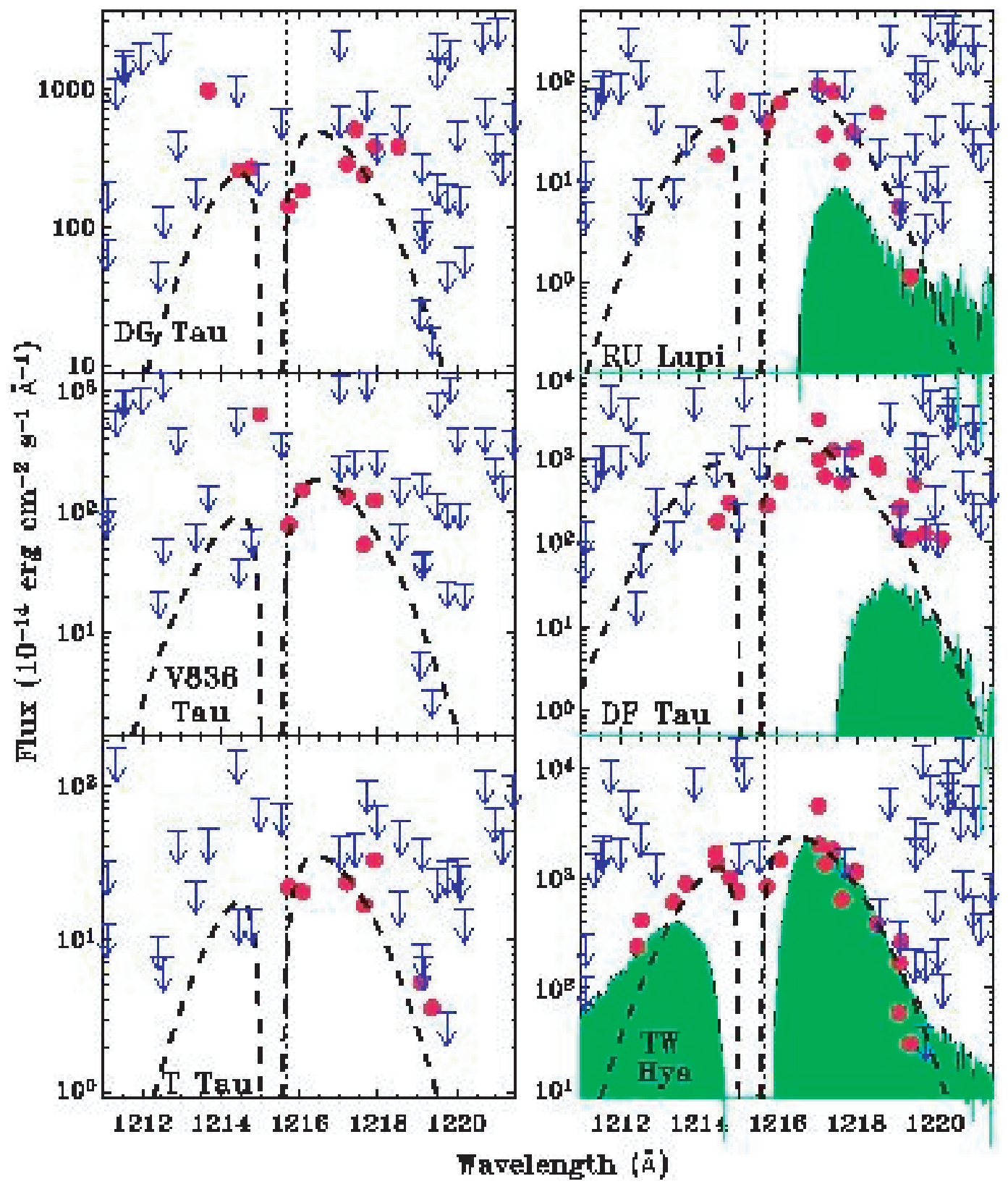

Figure 5. Reconstructed Ly $\alpha$ line profiles for the six TTSs. The intrinsic Ly $\alpha$ emission at many pumping wavelengths (solid circles) is calculated using the detected $\mathrm{H}_{2}$ emission from each pumped upper level, the effective equivalent width of the plane-parallel slab, and the estimated volume filling factor of the warm $\mathrm{H}_{2}$ gas as seen by the Ly $\alpha$ emission region. Both the observed and reconstructed fluxes shown here are dereddened. Upper limits (arrows) are calculated using upper limits of the total flux from each Ly $\alpha$-pumped $\mathrm{H}_{2}$ upper level. For RU Lupi, DF Tau and TW Hya, we compare the reconstructed to the observed Ly $\alpha$ emission (shaded). The vertical dotted lines indicate the stellar photosphere rest wavelength of $\mathrm{Ly} \alpha$. The dashed lines are the combination of a Gaussian emission and a central absorption profile fit to the reconstructed Ly $\alpha$ profile of TW Hya, scaled to match the reconstructed Ly $\alpha$ flux points for each star.

The $\mathrm{H}_{2}$ flux from the upper level is corrected for dissociation and the angular dependence of the photons escaping the disk. The integral is the effective equivalent width of the pumping transition for a plane-parallel slab with temperature $T$ and column density $N\left(\mathrm{H}_{2}\right)$. We assume that the disk geometry and gas properties used by Herczeg et al (2004) to model $\mathrm{H}_{2}$ fluorescence from TW Hya, in particular $T=$ $2500 \mathrm{~K}$ and $\log N\left(\mathrm{H}_{2}\right)=18.5$, apply to our entire sample. Any difference in the incidence angle between the incoming radiation and the disk surface will only change the effective column density. Figure 4 shows the ranges of temperature and $\mathrm{H}_{2}$ column density in the disk that are consistent with this analysis.

\section{Ly $\alpha$ lines in other PMS stars and ionization of disks}

Herczeg et al (2008) are now analyzing the fluorescent $\mathrm{H}_{2}$ spectra of six PMS stars observed by STIS and reconstructing their $\mathrm{Ly} \alpha$ line profiles. We report here on our preliminary analysis of these data. Figure 5 shows that portions of the red wings of two PMS stars, RU Lup and DF Tau are detected, but no portions of the Ly $\alpha$ profiles of the other three stars, DG Tau, V836 Tau, and T Tau, are detected. Using the method previously described, we have calculated the intrinsic Ly $\alpha$ emission and upper limits at the pumping wavelengths. This analysis allowed us to compute the total $\operatorname{Ly} \alpha$ flux and to 


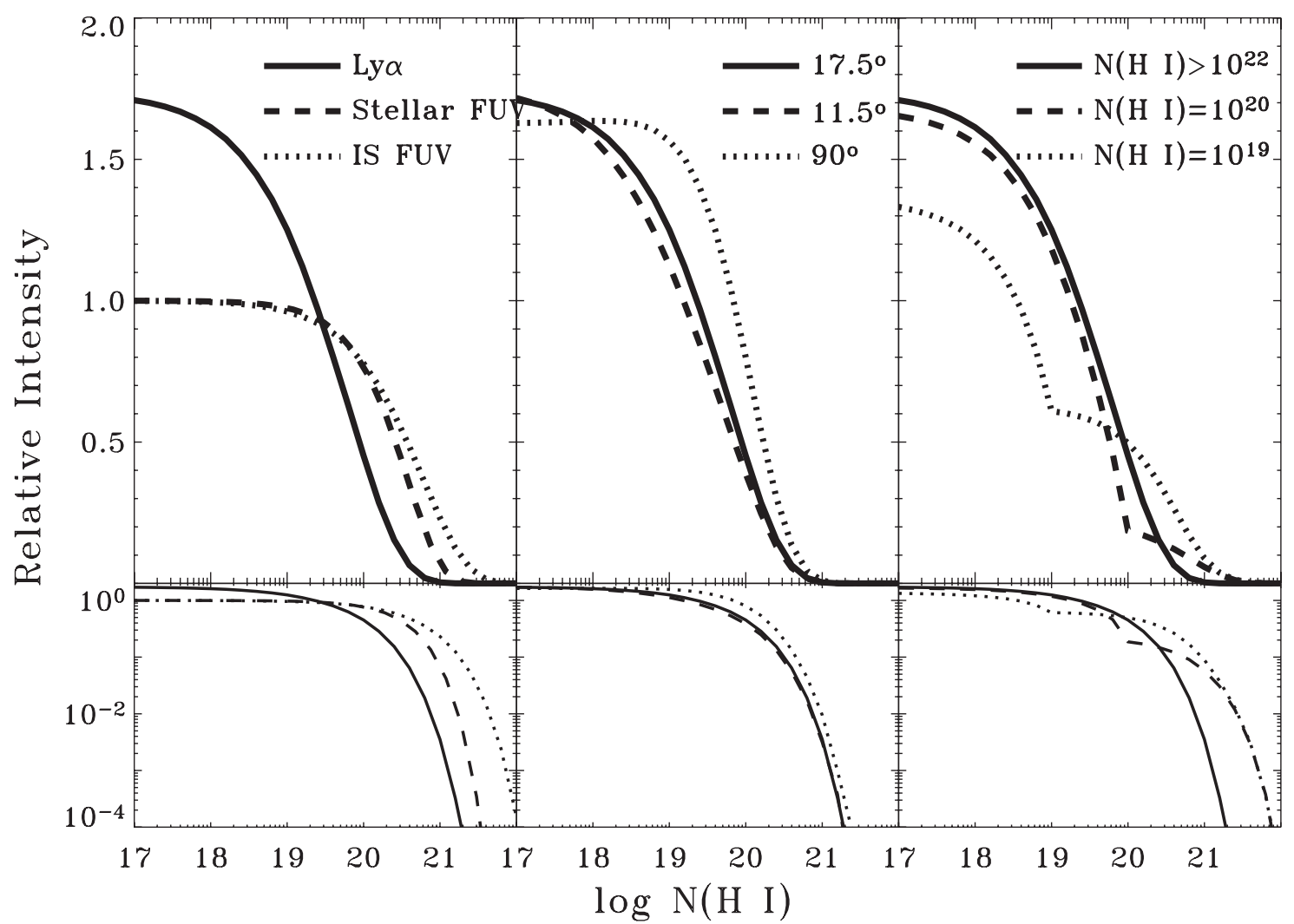

Figure 6. Relative intensity of Ly $\alpha$ emission in the disk as a function of neutral hydrogen column density $N(\mathrm{H} \mathrm{I})$, measured perpendicularly inwards from the disk surface. Left: scattering by $\mathrm{H}$ I amplifies the Ly $\alpha$ intensity at $\log N(\mathrm{H})=\log \left[N(\mathrm{H} \mathrm{I})+2 N\left(\mathrm{H}_{2}\right)\right]<20$, but with increasing path length, stellar Ly $\alpha$ photons do not penetrate as far into the disk as non-Ly $\alpha$ FUV photons. Centre: the penetration depth of Ly $\alpha$ into the disk is similar for an entrance angle of $11.5^{\circ}$ at $10 \mathrm{AU}$ and $17.5^{\circ}$ at $100 \mathrm{AU}$. Right: the Ly $\alpha$ emission may be less amplified but penetrate to larger $N(\mathrm{H})$ when the total $N(\mathrm{H} \mathrm{I})$ column density at the disk surface is small, which could happen if the $\mathrm{H} / \mathrm{H}_{2}$ transition occurs close to the disk surface. The three curves are for models where the $\mathrm{H} / \mathrm{H}_{2}$ transition occurs at different values of $N(\mathrm{H} \mathrm{I})$. In each model, the Ly $\alpha$ emission irradiating the disk was estimated from the reconstructed Ly $\alpha$ profile of TW Hya.

compare it with the other UV emission lines and continua. We find that the reconstructed Ly $\alpha$ line contributes $75-90 \%$ of the total UV emission for three of the stars (TW Hya, DF Tau and V836 Tau) but only $10-15 \%$ of the total UV flux for RU Lupi and T Tau.

The bright Ly $\alpha$ line plays an important role in the ionization and heating of the outer layers of the circumstellar disks of PMS stars. Figure 6 shows that Ly $\alpha$ dominates the input ionizing flux down to a column depth of $\log N(\mathrm{H} \mathrm{I}) \approx 20$ under a variety of conditions. Figure 7 shows that at a distance of $30 \mathrm{AU}$ from TW Hya, Ly $\alpha$ and stellar FUV radiation are more important than interstellar radiation, which is incident from all directions, to even larger hydrogen column densities measured from the surface of the circumstellar disk.

\section{Summary}

(i) Although the strong Ly $\alpha$ line is mostly or completely hidden from view in CTTSs, we describe a method for reconstructing the Ly $\alpha$ line from the observed fluorescent $\mathrm{H}_{2}$ emission.

(ii) The reconstructed Ly $\alpha$ emission contributes between 75 and $90 \%$ of the total FUV emission from TW Hya, DF Tau and V836 Tau, but only $10-15 \%$ of the total FUV emission from RU Lupi and T Tau. This difference results from the much higher effective filling factor for RU Lupi relative to TW Hya and DF Tau. Since the

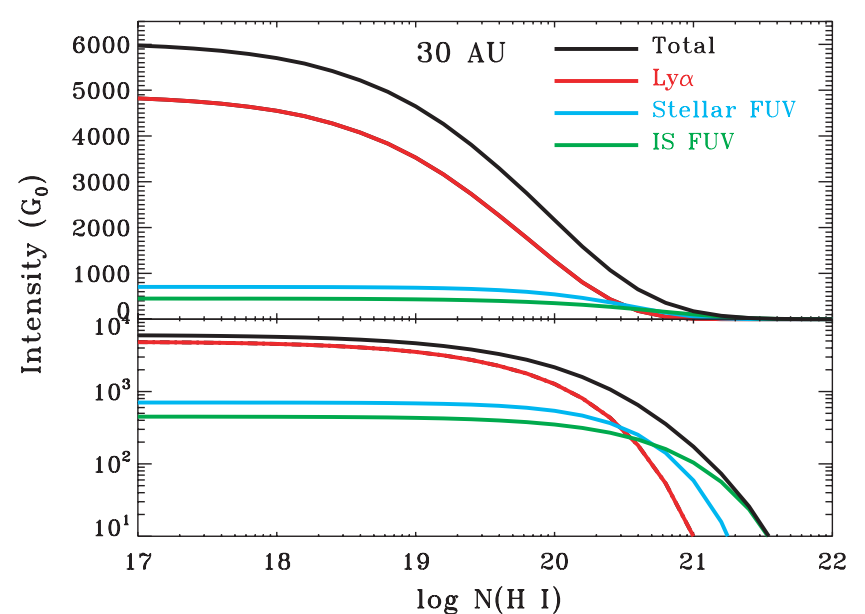

Figure 7. The total FUV radiation field (black line) in the disk versus $N(\mathrm{H} \mathrm{I})$ measured inward from the disk surface at $30 \mathrm{AU}$ from TW Hya. The FUV radiation incident on the disk is a combination of stellar Ly $\alpha$ photons (red line) and other stellar FUV photons (blue line), which strike the disk at $\alpha=17.5^{\circ}$, and interstellar FUV photons (green line), which enter the disk at all angles. The Ly $\alpha$ radiation penetrates to larger $\mathrm{N}(\mathrm{H} \mathrm{I})$ when the $\mathrm{H} / \mathrm{H}_{2}$ transition occurs at $\log N(\mathrm{H} \mathrm{I})=19.0$ (right), relative to models where the transition occurs at larger $\log N(\mathrm{H} \mathrm{I})$.

$\mathrm{H}_{2}$ emission from RU Lupi is produced by outflows, we attribute the different effective filling factors to the different geometries. 
(iii) The lower limit of the total FUV emission ranges from $3 \times 10^{-3} L_{\odot}$ to $3 \times 10^{-2} L_{\odot}$. The total Ly $\alpha$ emission depends on the effective filling factor of $\mathrm{H}_{2}$ as seen by the Ly $\alpha$-emitting gas.

\section{Acknowledgments}

This work is funded by Space Telescope Science Institute grant AR-9930 to the University of Colorado. This work is based on observations made in programs 7718, 8041 and 8157 (PIs J L Linsky and F M Walter) of the NASA/ESA Hubble Space Telescope, obtained at the Space Telescope Science Institute, which is operated by the Association of Universities for Research in Astronomy, Inc., under NASA contract NAS5-26555.

\section{References}

Abgrall H, Roueff E, Launay F, Roncin J Y and Subtil J L 1993 Astron. Astrophys. Suppl. $101273-321$

Herczeg G J, Linsky J L, Valenti J A, Johns-Krull C M and Wood B E 2002 Astrophys. J. 572 310-25

Herczeg G J, Wood B E, Linsky J L, Valenti J A and Johns-Krull C M 2004 Astrophys. J. 607 369-83

Herczeg G J, Linsky J L, Walter F M, Gahm G F and Johns-Krull C M 2006 Astrophys. J. Suppl. $165256-82$

Herczeg G J, Linsky J L and Wood B E 2008 in preparation

Johns-Krull C, Valenti J A and Linsky J 2000 Astrophys. J. 536 $815-33$

Valenti J A, Johns-Krull C and Linsky J L 2000 Astrophys. J. Suppl 129 399-420 